\title{
Determinación de la Eficiencia de Campo para el Sistema de Máquinas de alza y transporte en el Ingenio Pantaleón utilizando diagramas cíclicos, en la zafra azucarera 2010/2011
}

\author{
R. Rivera ${ }^{\ddagger}$ N. Sánchez, G. Ríos \\ Facultad de Tecnología de la Construcción, Universidad Nacional de Ingeniería (UNI) \\ PO Box 5595, Managua, Nicaragua. \\ e-mail: rrivera 63@yahoo.com; sancheznorwin@hotmail.com; gerardorios887@yahoo.com
}

(Recibido/received: 08-Mayo-2012; aceptado/accepted: 04-Octubre-2012)

\section{RESUMEN}

La ingeniería agrícola relacionada con los procesos mecanizados, no se limita a estimar sólo la operación de las máquinas agropecuarias en un estado fijo del sistema, sino que trata de predecir el estado exacto del sistema en cualquier instante, no solo con el objetivo de identificar las actividades de todo el sistema de máquinas, sino también encontrar las eficiencias de campo del sistema, la eficiencia de mano de obra y además, realizar un análisis de los tiempos de los ciclos en tiempo real.

Este estudio permitió determinar -a nivel piloto-, en la práctica las eficiencias de campo; mano de obra, el rendimiento de las maquinas, así como identificar los factores que restringen el sistema de máquinas en el proceso de Alza y Transporte de Cosecha de la caña de azúcar en el Ingenio Pantaleón, ubicado en El Viejo, Chinandega, Nicaragua.

Palabras clave: eficiencia de campo; rendimiento de máquina; sistemas de cosecha mecanizados.

\begin{abstract}
The agricultural engineering, related to the mechanized processes, is not limited to estimate the machines performance in a fixed state of the system. Indeed, it tries to predict the exact state of the system at any time with the purpose of identifying the activities of the machine system, and find the field efficiencies of the system, and labor efficiency. Furthermore, conduct an analysis of the cycle times in real-time.

This study helped to find in practice, at a pilot level, the field efficiencies; labor efficiency, and the machines performance. Moreover, we identified the factors that restrict the machine system in the process of rise and transport of sugar cane harvest of Ingenio Pantaleón in El Viejo, Chinandega, Nicaragua
\end{abstract}

Keywords: Field efficiency; machine performance; mechanized harvesting systems.

${ }^{*}$ Autor para la correspondencia 


\section{R. Rivera, N. Sánchez y G. Ríos}

\section{INTRODUCCIÓN}

En la agricultura y en especial en el campo de la mecanización agropecuaria es común encontrar dos tipos de operaciones mecanizadas: a) Secuenciales y b) Paralelas. (D. Hunt, 1983)

La ingeniería agrícola relacionada con los procesos mecanizados, no se limita a estimar sólo la operación de las máquinas agropecuarias en un estado fijo del sistema, sino que trata de predecir el estado exacto del sistema en cualquier instante, no solo con el objetivo de identificar las actividades de todo el sistema de máquinas, sino también encontrar las eficiencias de campo del sistema, la eficiencia de mano de obra y además, realizar un análisis de los tiempos de los ciclos en tiempo real.

Uno de los problemas menos abordados en los ingenios azucareros es el mejoramiento de la eficiencia de los frentes de cosecha mecanizados y por consiguiente la disminución del desabastecimiento de caña a granel desde los centros de recolección hasta la fábrica. Esta situación, es magnificada por el hecho de la inexistencia de metodologías aplicadas a la agricultura que permitan analizar tiempos de trabajo.

Un Diagrama Cíclico es un método de apoyo para el análisis de un sistema de máquinas de campo; su utilidad radica en que estos diagramas indican la actividad de todo el sistema de máquinas. Estos son construidos en base a la aplicación de técnicas de medición del trabajo, los cuales registran los tiempos y ritmos de las labores correspondiente a los elementos de una tarea definida, permitiendo posteriormente el análisis de los datos a fin de averiguar el tiempo requerido para efectuar la tarea según una norma establecida. (Kanawaty, G., 2004)

Este estudio permitió determinar -a nivel piloto-, en la práctica las eficiencias de campo; mano de obra, el rendimiento de las maquinas, así como identificar los factores que restringen el sistema de máquinas en el proceso de Alza y Transporte de Cosecha de la caña de azúcar, Ingenio Pantaleón, ubicado en el municipio de El Viejo, departamento de Chinandega.

\section{METODOLOGÍA}

En el presente estudio, se utilizó la metodología de Investigación de Campo, la cual es recomendada cuando la misma se efectúa en el lugar y tiempo en que ocurren el o los fenómenos objeto de estudio
Inicialmente, se realizó un análisis documental con la finalidad de: 1) obtener un amplio conocimiento del tema relacionado con el proceso de cosecha de los frentes mecanizados utilizados en el Ingenio Pantaleón, $y, 2)$ adquirir información suficiente y ordenada para identificar los ciclos de trabajo de cada una de las máquinas que integran el sistema de cosecha, para ello se consultaron fuentes de información primarias y secundarias tales como: consulta de bases de datos electrónicas, informes, reportes, entrevistas al personal técnico del ingenio, docentes y especialistas en el área de Sistemas mecanizados que trabajan en Paralelo. Dentro de esta fase preparatoria, también se coordinó con las autoridades del Ingenio Pantaleón el trabajo para asegurar el apoyo logístico requerido.

El trabajo de campo, se inició con la recolección y toma de datos se llevó a cabo con el propósito de acumular y disponer datos suficientes de los tiempos y ciclos de trabajo de las maquinas durante el periodo zafra 2010/2011. En dicha etapa se alcanzó la cantidad de datos previstos en el cálculo del tamaño de la muestra. La determinación la misma, fue estimada por las ecuaciones 1 y 2 de la teoría de Muestreo. (Jany, J., 2005)

$$
\begin{aligned}
& \mathrm{n}=\frac{\mathrm{n}_{\mathrm{o}}}{1+\frac{\mathrm{n}_{\mathrm{o}}}{\mathrm{N}}} \\
& \mathrm{n}_{0=\frac{\mathrm{Z}^{2} \mathrm{~S}^{2}}{\mathrm{e}^{2}}}
\end{aligned}
$$

En las fases de análisis de datos, se utilizaron los procedimientos de Estadística descriptiva (J. Rocha. 2010), con el objetivo de procesar los conjuntos y masas de datos por medio de tablas, gráficos y otras medidas de resumen. La estadística descriptiva fue la primera etapa desarrollada para el análisis de la información.

La necesidad de una oportunidad comparativa de las maquinas individuales requiere de un enfoque diagramático para una estimación del rendimiento del sistema. El Diagrama Cíclico (Ver. Fig. 1) es un método de apoyo para el análisis de un sistema de máquinas en el campo trabajando en paralelo, estos diagramas indican la actividad de todo el sistema de máquinas. (D. Hunt, 1983)

Durante la zafra 2010/2011 del Ingenio Pantaleón se contó con cuatro frentes de cosecha mecanizados, los cuales están compuestos de las máquinas y equipos que se describen en la Tabla. 1. 


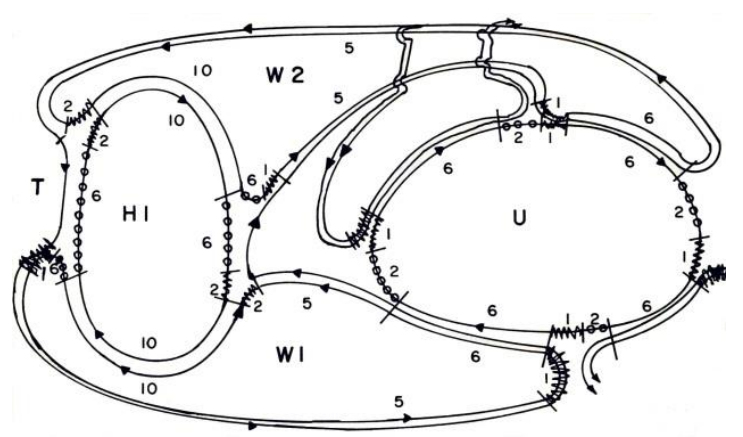

Figura 1 Ejemplo de un diagrama cíclico de un sistema de cosecha. (D. Hunt, 1983)

Tabla. 1 Especificaciones técnicas de las máquinas de un frente de cosecha mecanizada

\begin{tabular}{|c|c|c|c|c|c|c|}
\hline \multicolumn{7}{|c|}{ Sistema de máquinas Ingenio Pantaleón } \\
\hline Descripción & Marca & Modelo & Motor & $\begin{array}{c}\text { Capacidad } \\
\text { de carga }\end{array}$ & $\begin{array}{c}\text { Vel. } \\
\text { cosecha/alce }\end{array}$ & Rendimiento \\
\hline Cosechadora & Case IH & A7000/A7700 & $300 \mathrm{cV}$ & & $20 / 9 \mathrm{~km} / \mathrm{hr}$ & $70 \mathrm{t} / \mathrm{hr}$ \\
\hline Alzadora & Cameco & SP2254/SP1800 & $\begin{array}{c}155 / 100 \\
\mathrm{hp}\end{array}$ & & $5 \mathrm{~km} / \mathrm{hr}$ & $55 / 40 \mathrm{t} / \mathrm{hr}$ \\
\hline Tractor & $\begin{array}{c}\text { New } \\
\text { Holland }\end{array}$ & T7-250 & $250 \mathrm{hp}$ & & & \\
\hline Tractor & $\begin{array}{c}\text { New } \\
\text { Holland }\end{array}$ & Tm-175 & $155 \mathrm{hp}$ & & & \\
\hline Camión & Freightliner & FDL & $\begin{array}{c}\text { Detroit } \\
450 \mathrm{hp}\end{array}$ & $350 \mathrm{t}$ & & \\
\hline Autovolteo & Cameco & & & $8 \mathrm{t}$ & & $33 \mathrm{t}$ \\
\hline Vagón & Cameco & Promesa/Fama & & $33 \mathrm{t}$ & & \\
\hline
\end{tabular}

El frente mecanizado funciona así: La cosechadora (Ver. Fig. 2) corta e impulsa la caña cortada desde el lote asignado y la deposita en los vagones del sistema de Autovolteos en forma paralela y simultánea. Cuando la cosechadora completa el llenado de los vagones, el autovolteo sale del lote y se dirige hacia el área de traspase, sitio en el cual, la caña cortada es transferida por los sistemas hidráulicos del autovolteo hacia los vagones de transporte. En este sitio, el camión de transporte desengancha los vagones vacíos que trae de la fábrica y procede a enganchar los vagones recién cargados que están en el campo. A partir de ahí, se inicia el recorrido de los camiones remolcadores de los vagones cargados con la caña cortada hacia la báscula de pesaje en la fábrica, finalizado esta operación, el proceso se reinicia de forma ininterrumpida. Para este sistema de cosecha, y, utilizando las eficiencias parciales teóricas de cada una de las máquinas que la componen al trabajar en paralelo, es posible estimar su Eficiencia Teórica en un 51\%.

Un Frente de Cosecha Mecanizado es operado por: Un supervisor, cuatro operadores de cosechadora, nueve operadores de tractores y dos enboletadores y tres operadores auxiliares.

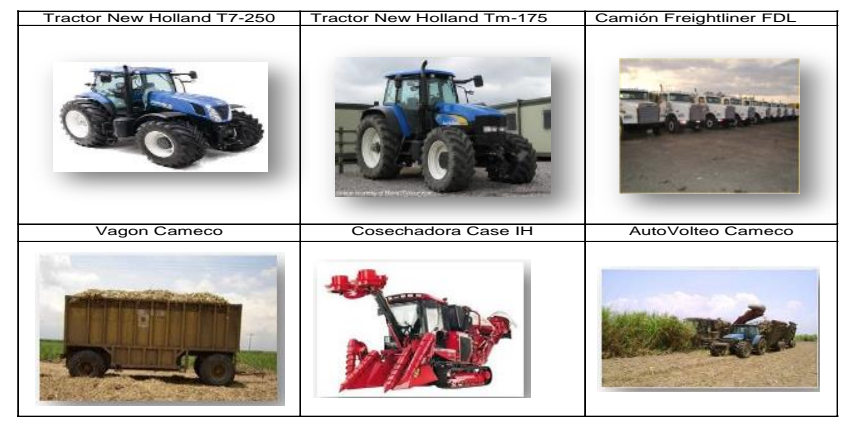

Figura 2. Maquinaria utilizada en un frente de cosecha mecanizada

En este estudio se utilizaron los siguientes recursos materiales, logísticos y de procesamiento de la información: Copias de formatos para la recolección de los tiempos, cronómetro digital, tablero de observaciones, hojas electrónicas Excel para el procesamiento de datos, Paquete Estadístico Statistics Package for the Social Sciences (SPSS) version 19, AutoCAD 2011, computadora portátil marca Acer modelo ASPIRE one.

\section{RESULTADOS Y DISCUSIÓN}

Una vez estimadas la cantidad de muestras requeridas para el estudio, se elaboraron los formatos para la recolección de los tiempos de trabajo, en función de los ciclos de trabajo de cada una de las máquinas que componen el Frente de cosecha mecanizado. Asimismo, se procedió a organizar de forma coordinada el trabajo logístico con las autoridades del departamento de Alza y Transporte del Ingenio, se procedió a levantar los datos de campo.

Finalizado el proceso de recolección de los datos de campo correspondientes al periodo de Zafra 2010/2011. Para procesar la información se utilizó el paquete estadístico, Statistics Package for the Social Sciences (IBM) (SPSS) (ver 19).Con esta herramienta informática, se procesaron las series de datos de tiempo. Se obtuvieron las medidas de tendencia central: Media $(\overline{\mathrm{X}})$, Moda (MO), Desviación Típica(S), valores Mínimos y Máximos, además de los datos de Distribución Normal(X), y el gráfico P-P.

Mediante la revisión de la normalidad de los datos de tiempo de enganche, se puede observar que los puntos siguen aproximadamente a la curva teórica, (Ver. Fig. 3) lo que indica que los datos se distribuyen de forma normal con una confianza del $95 \%$. Esta normalidad se analizó para tener la confianza de que la estimaciones 


\section{R. Rivera, N. Sánchez y G. Ríos}

que se hicieron son válidas ya que se le puede distribuir normalmente (Ampié J, 2011)

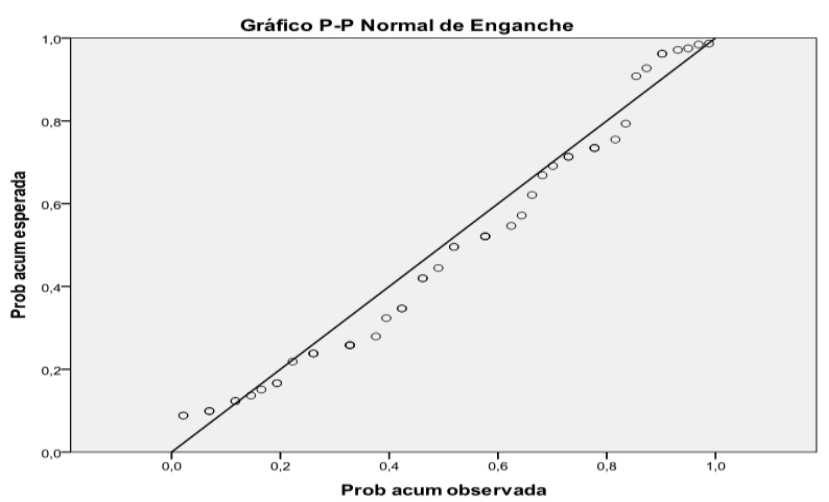

Figura 3 Gráfico P-P Curva normal del tiempo de enganche

Fueron determinados y analizados los eventos de todos los ciclos individuales para cada una de las máquinas que conforman el sistema de máquinas del frente de cosecha. Un detalle de los resultados obtenidos es presentado en la Tabla 2.

Tabla. 2 tiempos medios del frente mecanizado

\begin{tabular}{|c|c|c|c|c|c|}
\hline \multicolumn{6}{|c|}{ Tiempos promedios (min) Frente mecanizado } \\
\hline $\begin{array}{c}\text { Evento } \\
\text { Equipo }\end{array}$ & Recorrido vacio & Desenganhe & Carga & Enganche & Recorrido cargado \\
\hline $\begin{array}{c}\text { Camion } \\
\text { Cosehadora }\end{array}$ & 46.7 & 1.9 & 17.8 & 4.0 & 58.8 \\
\hline Autovolteo & 3.1 & & 22.8 & & 3.1 \\
\hline
\end{tabular}

A partir de los datos de la tabla anterior se procedió al cálculo de los tiempos ociosos (tiempos muertos) para el frente de cosecha mecanizada. Y con los valores obtenidos se elaboró en AutoCAD 2011 el Diagrama Cíclico del Sistema, el cual se muestra en la Figura 4.

Fue calculada, la capacidad del sistema en estado fijo para el sistema mecanizado, obteniendo un valor de 11,6 cargas/hora $[1,914 \mathrm{t} / \mathrm{h}]$. La eficiencia de la mano de obra, con un trabajador para cada máquina, fue de 0,6 cargas/hr._hombre.

Si se emplea una eficiencia de campo del $70 \%$, para una cosechadora de caña (D. Hunt, 1983), del total del tiempo de 22,8 minutos, estos se descompondrían en 15,96 minutos de tiempo teórico de trabajo efectivo en el campo, y 6,84 minutos de tiempo de apoyo (tiempos de virajes, ajustes de la máquina, mantenimiento de campo y reparaciones), e incluyendo los 83,6 minutos de tiempo ocioso, se encontró que la eficiencia de campo real del sistema fue de:

\section{$15,96 /(15,96+6,84+83,6) \cong 15 \%$}

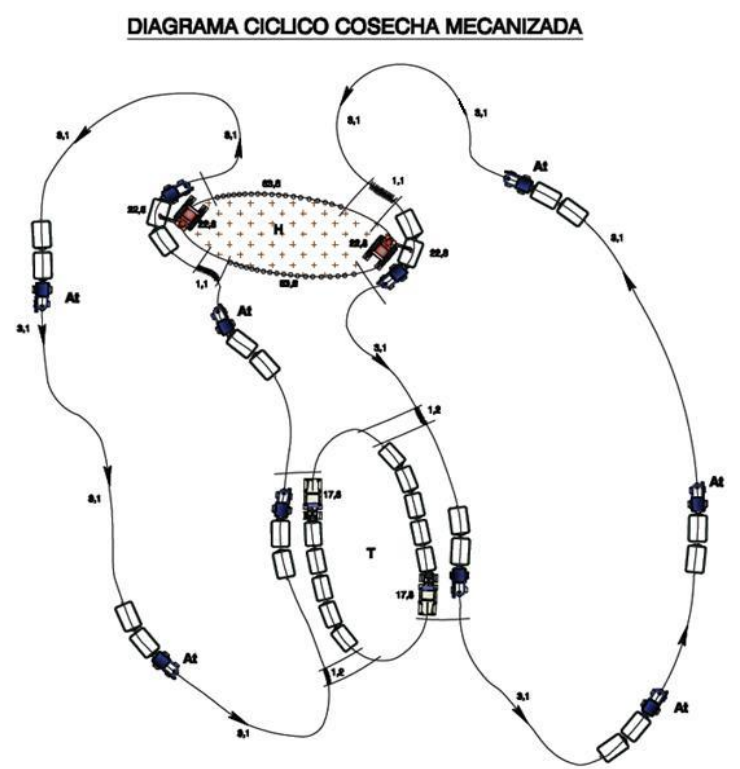

Figura 4 Diagrama Cíclico del Frente Mecanizado (Fuente Propia)

Se define el Error Absoluto como la diferencia entre el valor de la medida aproximada y el valor tomado como exacto. El Error Relativo se define como el cociente entre el valor absoluto y el valor exacto. Para medir la magnitud del error del sistema mecanizado, y, asumiendo como valor exacto, el obtenido de la Eficiencia teórica esperada para el sistema, la cual resulta de la productoria de las eficiencias individuales de cada máquina que componen el sistema (Ashbuner \& Sims. B, 1984), y, como valores aproximados, los valores medios, moda y mínimos obtenidos a partir de la estadística descriptiva utilizada en el análisis, los cuales se resumen en la Tabla 3.

Tabla 3 Errores absolutos y relativos de cada medida

\begin{tabular}{|c|c|c|c|}
\hline Medida & Errores Absolutos & Errores Relativos & En Porcentaje \\
\hline Medio & 0.36 & 0.71 & $71.0 \%$ \\
\hline Moda & 0.41 & 0.81 & $81.0 \%$ \\
\hline Mínimo & 0.06 & 0.11 & $11.0 \%$ \\
\hline
\end{tabular}

De la tabla anterior se observa, que cuando se utilizan los tiempos mínimos en el cálculo de las eficiencias del sistema, el error se reduce significativamente hasta en un $6 \%$ (ea), y en un $11 \%$ (er). La eficiencia alcanzada 
(45.5\%), cuando se utilizan los tiempos mínimos no sobrepasa la eficiencia teórica del sistema, esperada del $51 \%$. Lo que indica que el Sistema de cosecha mecanizada trabajando en paralelo, mejora notablemente si se minimizan los tiempos ociosos los cuales los hacen poco efectivo y desabastecen el transporte de caña de azúcar a granel desde las áreas de recolección hasta la fábrica. Este análisis permite precisar que el proceso es menos eficiente en el área de transferencia, que es donde los camiones desenganchan y enganchan los vagones procedentes de la báscula de la fábrica al campo.

\section{CONCLUSIONES}

El método del Diagrama Cíclico resulta ser pertinente y eficaz para el análisis de un sistema de máquinas trabajando en paralelo, como es el caso de la cosecha mecanizada de la caña de azúcar. La cantidad y calidad los datos recolectados en el campo, una vez analizados obtuvieron una confiabilidad del $95 \%$.

Se determinó que la eficiencia (15\%) del Sistema Mecanizado, está muy por debajo de la eficiencia teórica esperada para un Sistema de Máquinas (51\%) trabajando en paralelo.

Cuando se utilizan valores de tiempos mínimos, el error relativo en términos porcentuales se aproxima favorablemente al valor teórico hasta en un $11 \%$, y en un $6 \%$ en el caso del error absoluto.

El equipo que limita la eficiencia del Frente Mecanizado, y genera un cuello de botella al mismo, es el área de traspase de los camiones transportadores que van desde el centro de recolección a la fábrica.

\section{AGRADECIMIENTOS}

Se les agradece a las autoridades y personal técnico del departamento de Alza y Transporte del Ingenio Pantaleón por brindar las facilidades y logísticas necesarias para llevar a cabo este estudio.

\section{REFERENCIAS}

Ampié, J (2010) Estadística Básica, Documento Guía ( $1^{a}$ Edición $)$ Ed. Departamento de Matemática, Recinto Universitario Pedro Arauz Palacios. Mangua Nicaragua.

Ashburner, J (1984) Elementos de Diseño del trabajo y Herramientas de Labranza. (2 ${ }^{a}$ edición) Ed. Instituto
Interamericano de Cooperación para la agricultura Costa Rica.

Hunt, D. (1983). Maquinaria Agrícola Rendimiento económico, costos operaciones potencia y selección de equipo. ( $7^{\text {a }}$ Edición) Ed. Limusa México.

Jany, J. (2005) Investigación Integral de Mercados. (3a edición) Mc Graw Hill Colombia.

Kanawaty, G. (2004).Introducción al estudio del trabajo. (4ª Edición) Ed. Limusa. México.

Rocha, J. (2010). Bioestadística, Maestría en salud Ocupacional ( $1^{\mathrm{a} E d i c i o ́ n) ~ E d . ~ C e n t r o ~ d e ~ I n v e s t i g a c i o n e s ~}$ y Estudio de la Salud. Universidad Nacional Autónoma de Nicaragua. Managua, Nicaragua.

Ricardo Rivera Medina. Se graduó de Ingeniero Agrícola en la Universidad Nacional de Ingeniería

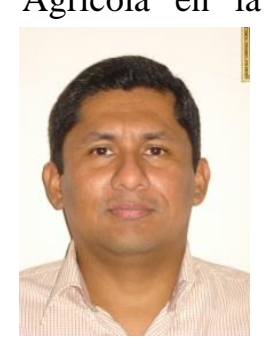
(UNI) en 1987. Se graduó de Doctor en la Universidad Politécnica de Catalunya (UPC) en 2003, en la especialidad de Oleohidráulica y Neumática. Es consultor en el área de mecanización agropecuaria y Profesor titular del departamento de Ingeniería Agrícola de la UNI.

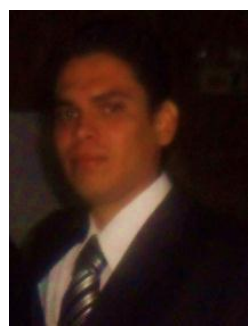

Norwin Sánchez Murillo. Se graduó de Ingeniero Agrícola en la Universidad Nacional de Ingeniería (UNI) en 2012. Su área de investigación es la mecanización agropecuaria con énfasis en los sistemas de cosecha mecanizados trabajando en paralelo

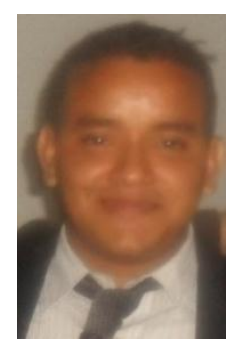

Gerardo Ríos Carrasco. Se graduó de Ingeniero Agrícola en la Universidad Nacional de Ingeniería (UNI) en 2012. Su área de investigación es la mecanización agropecuaria con énfasis en los sistemas de cosecha mecanizados trabajando en paralelo (UNI) en 2012. $\mathrm{Su}$ área de investigación es la mecanización agropecuaria con énfasis en los sistemas de cosecha mecanizados trabajando en paralelo. 